\title{
TRANS-
}

Revue de littérature générale et comparée

Informations

\begin{tabular}{|c|c|c|c|c|}
\hline & & CATALOGUE & & Tout \\
\hline $\begin{array}{l}\text { OpenEdition } \\
\text { Journals }\end{array}$ & ACCUEIL & $\begin{array}{l}\text { DES } 546 \\
\text { REVUES }\end{array}$ & OPENEDITION SEARCH & OpenEdition \\
\hline
\end{tabular}

Titre : TRANS-

Sous-tittre : Revue de littérature générale et comparée

Éditeur : Centre d'Études et des Recherches Comparatistes de l'Université

Sorbonne nouvelle - Paris 3 .

Directeur de la publication : Yen-Mai Tran-Gervat

Responsable de l'édition électronique : Iván Salinas Escobar

Type de support : Électronique

Politique d'édition électronique : Publication intégrale en libre accès

Périodicité : Semestrielle

Année de création : 2005

ISSN format électronique : $1778-3887$ 


\title{
TRANS-
}

Revue de littérature générale et comparée

Des copies originales $\left(\mathrm{N}^{\circ} 22 \mid 2017\right)$

Traducteurs, copieurs originaux

\section{Traduire un texte entre les langues. Retour sur l'expérience de traduction de Hong bai hei}

\author{
Paolo Magagnin
}

\section{Résumés}

Français English

Cette contribution se propose d'explorer la stratégie de conservation et de recréation des éléments d'hétérogénéité dans la traduction italienne du roman Hong bai hei ( Blanc et le Noir ») de l'auteur sino-canadien Chen He. À sa première parution en 2007, le roman a été salué comme un exemple d'écriture fortement " globale »: en effet, il est caractérisé par une forte hétérogénéité au niveau des thèmes, des pays où l'action se déroule, des langues utilisées, des genres et des sources exploitées. Dans le processus de traduction, cette pluralité d'éléments appelle naturellement à une stratégie minorisante qui viserait à reproduire, dans la plus ample mesure possible, l'altérité intrinsèque de l'original. Toutefois, le traducteur a également mis en place une stratégie de recréation du texte premier, qui s'est réalisée dans l'insertion originale d'éléments hétérolingues et dans l'interaction avec l'auteur afin de recréer des passages localisés du roman. En dernière analyse, cette stratégie de traduction créatrice et « globalisante » contribue à la redéfinition du texte premier et à la mise en relief de sa nature hétérogène et globale.

The aim of this paper is to investigate the strategy adopted for the conservation and recreation of heterogeneous elements in the Italian translation of Hong bai hei ("The Red, the White and the Black") by the Chinese-Canadian author Chen He. Upon its first publication in 2007, the novel was praised as an example of powerfully "global" writing: as a matter of fact, it is characterized by a high degree of heterogeneity at the level of themes, settings, languages, genres, and sources. In the translation process, this plurality of elements naturally calls for a minoritizing strategy aiming to reproduce, to the highest possible extent, the intrinsic heterogeneity of the original. However, the translator also carried out a strategy that points to the recreation of the original text: it took the form of a deliberate insertion of heterolinguistic elements, as well as an interaction with the author in order to recreate specific passages of the novel. In the final analysis, such a creative and "globalizing" strategy helps redefine the original text and highlight its heterogeneous and global nature. 


\section{Texte intégral}

Chen He 陈河 (pseudonyme de Chen Xiaowei 陈小卫), né à Wenzhou en 1958, commence sa carrière d'écrivain vers le milieu des années 80. En 1994 il s'installe à Tirana, où il s'adonne au commerce de produits pharmaceutiques et ensuite à la gestion d'un restaurant, avant de repartir au Canada en 1999 suite au déclenchement des révoltes albanaises. Après s'être établi à Toronto, il poursuit une activité commerciale pendant quelques années, avant de se consacrer définitivement à l'écriture romanesque en 2005.

Zhiming de yuanxing 致命的远行 ( Un long voyage fatal ») paraît en l'automne 2007 dans un numéro spécial de la revue littéraire Shouhuo 收获 ( La Moisson »), avant d'être republié en 2012 sous le titre Hong bai hei 红白黑 (L »e Rouge, le Blanc et le Noir »). Le roman suit les péripéties de Xie Qing, simple chauffeur de camions d'AC (sigle derrière lequel se cache à peine la ville de $\mathrm{Wenzhou}^{1}$ ), qui en 1993 est convoqué d'urgence à Paris pour reconnaître le corps de sa femme Yang Hong, décédée dans un mystérieux accident de voiture. Déterminé à enquêter sur le passé récent de sa femme, dont il n'avait plus de nouvelles depuis des années, Xie Qing décide de rester à Paris. Il fait ensuite la connaissance de sa compatriote Huang Qiumei, charismatique patronne de restaurant et trafiquante d'êtres humains, qui lui offre de participer à ses affaires illicites en l'envoyant en Italie, en Grèce et finalement en Albanie. Après l'échec tragique d'un passage de clandestins vers l'Italie, il rentre précipitamment à Paris, où il prend en charge les restaurants de Qiumei, qui a entretemps trouvé refuge en Tunisie. Le succès de Xie Qing dans la communauté chinoise parisienne le ramène bientôt à $\mathrm{AC}$ où, grâce à ses connaissances politiques, il fonde un empire immobilier florissant. L'arrestation de Qiumei et son internement dans un camp de travail du Qinghai porte pourtant un coup décisif à ses affaires, le poussant à rentrer en France en faisant profil bas.

Parallèlement, la narration suit en flashback l'enfance de Xie Qing à partir du début des années 60, sa rencontre avec Yang Hong et les événements menant à leur mariage malheureux sur la toile de fond des événements qui agitent la Chine, du chaos de la Révolution culturelle jusqu'aux reformes post-maoïstes. Le récit se focalise ensuite sur Yang Hong : ses enquêtes sur le passé énigmatique de son père, fonctionnaire du Parti communiste s'étant suicidé pendant la Révolution culturelle, l'amènent à entrer en contact avec un groupe de fils d'influents fonctionnaires pékinois déterminés à poursuivre les idéaux révolutionnaires de leurs pères. Leur chef, Jiang Xiaojun, la persuade de le suivre à Paris. En France, tous deux deviennent amants : ils ont un enfant, et Yang Hong entame une petite activité commerciale dans une localité côtière. Elle semble avoir enfin trouvé sa stabilité, mais quand Jiang est obligé de rentrer en Chine et qu'il rejoint sa famille chinoise, elle tombe dans un profond désarroi qui la conduit à la mort. La fin du roman voit Xie Qing rencontrer Jiang Xiaojun et l'enfant devant le tombeau de Yang Hong le jour de Qingming - la fête des morts de la tradition chinoise - de 2003.

\section{L'hétérogénéité entre langues, thèmes, genres, sources}

La caractéristique la plus évidente du roman, qui émerge déjà de son intrigue, est la mise en scène d'une hétérogénéité qui se manifeste à plusieurs niveaux. D'abord, l'altérité passe par la description de pays différents - Chine, France, Grèce, Albanie, Italie, Tunisie - et d'espaces hybrides et déplacés de par leur nature même - la Chinatown parisienne de Belleville, la communauté criminelle de Tirana où, à côté ou 
en concurrence avec les albanais, opèrent également des trafiquants provenant des autres pays balkaniques, italiens, chinois, etc. L'altérité territoriale ne se réalise pas qu'au niveau international, mais se traduit également dans des réflexions sur la distance entre la dimension provinciale d'AC/Wenzhou et celle de la capitale Pékin.

L'hétérogénéité s'incarne encore dans des personnages à la psychologie complexe et exposée à des influences culturelles multiples, déracinés de leur milieu et plus ou moins intégrés dans un autre contexte auquel ils sont constamment confrontés. À côté des Wenzhounais de Paris comme Xie Qing et Huang Qiumei (qui par la suite achète une ferme tunisienne, apprend l'arabe et adopte même les habitudes vestimentaires locales) et des Pékinois fascinés par le style de vie et la pensée occidentaux comme Jiang Xiaojun, on retrouve un Hongkongais à Athènes, des Italiens en Albanie, des Chinois émigrés à Rome, à Tirana, à Vienne et à Munich, un Albanais ayant fait ses études à Hangzhou, et ainsi de suite.

Le roman est caractérisé aussi par d'importants éléments hétérolingues : en effet, à côté du chinois mandarin on observe la présence de nombreux lexèmes propres au dialecte de Wenzhou transcrits en caractères chinois (dont la prononciation en mandarin standard ne correspond pourtant pas à leur réalisation effective en wenzhounais, et qui sont seulement parfois accompagnés d'une glose) et, dans une mesure plus réduite, de mots ou phrases entières en français, anglais, albanais et italien (transposés soit directement en lettres latines, soit dans une transcription approximative en caractères chinois).

Un autre signal d'hétérogénéité est constitué par les nombreuses réflexions métaculturelles (quoique souvent stéréotypées) et métalinguistiques au bénéfice d'un public chinois indifférencié, qui représente la cible principale de l'auteur ${ }^{2}$. Outre des annotations expliquant certaines références culturelles non chinoises, l'auteur ajoute des observations sur le contexte chinois en général, ainsi que des commentaires sur la spécificité socioculturelle de la région d'AC/Wenzhou qui paraissent s'adresser plutôt à un public international, comme dans les extraits suivants :

在巴黎的 $\mathrm{AC}$ 人大概有十万, 有七万多集中在这几个区份。 $\mathrm{AC}$ 人在中国是一种很 边缘的群体, 既像犹太人那样精于做生意, 也像吉普赛人一样喜好到处流浪3。

À Paris vivaient environ cent mille AÇois, dont soixante-dix mille concentrés dans ces quartiers. En fait, en Chine, les AÇois représentent une communauté plutôt marginale : comme les juifs ils ont un don pour les affaires, et comme les gitans ils aiment vagabonder d'un lieu à l'autre.

她越来越不喜欢夜郎自大的 $\mathrm{AC}$ 本地人了。她发现, $\mathrm{AC}$ 人像井底之蛙, 老是觉得 $\mathrm{AC}$ 这个地方是领导世界潮流的中心，到处在吹嘘什么 $\mathrm{AC}$ 模式 $\mathrm{AC}$ 现象 4 。

Les AÇois, avec leur esprit de clocher arrogant, lui étaient de plus en plus insupportables : elle se rendit compte qu'ils se comportaient comme la grenouille au fond du puits, ils étaient convaincus qu'AC était le centre du monde et toujours prêts à faire l'éloge du « modèle $\mathrm{AC}$ », du « phénomène $\mathrm{AC}$ » et ainsi de suite.

Finalement, hétérogénéité et hybridité émergent sur le plan des genres et des sources exploitées par l'auteur. Si l'emploi des formes expressives et des tropes du roman noir d'inspiration occidentale est évident au début de la narration, ceux-ci s'accompagnent bientôt de l'émergence de modèles littéraires essentiellement chinois : surtout la tradition de la littérature (mémorialiste et fictionnelle) sur la période de la Révolution culturelle, mais également certains éléments du roman d'anti-corruption ou fanfubai xiaoshuo 反腐败小说 (dans la description des magouilles auxquelles se livre la classe politique locale d'AC) et même du roman de cape et d'épée ou wuxia xiaoshuo 武侠小说 (p. ex. l'insistance sur le code d'honneur et de loyauté inébranlable qui lie le « disciple » Xie Qing à son « maître » Huang Qiumei).

À ces facteurs s'ajoute la composante autobiographique dont le roman se nourrit copieusement : non seulement l'enfance et la jeunesse de Xie Qing calquent 
largement le vécu de l'écrivain lui-même, mais la biographie de ce dernier offre une source d'inspiration indéniable à d'autres sections du récit, comme l'expérience de Xie Qing en tant que chauffeur routier, ou l'épisode du kidnapping dont il est victime en Albanie $^{5}$.

Le titre même du roman mérite une attention spéciale, en tant que jeu d'hybridation qui mélange la citation stendhalienne à des connotations culturelles essentiellement chinoises. Le lien intertextuel immédiat avec Le Rouge et le Noir, en effet, semble suggérer une certaine affinité entre le personnage de Xie Qing et l'ambitieux Julien Sorel. Cependant, les couleurs évoquées dans le titre chinois sont porteuses d'implications particulières, puisqu'elles font référence aux trois axes autour desquels se déploient l'intrigue et les parcours des personnages - à savoir la « voie rouge » (hongdao 红道), qui symbolise l'élément politique, la "voie blanche » (baidao 白道), qui se réfère à l'univers commercial et des affaires (mais également à celui des hors-laloi suivant un idéal de justice), et la « voie noire » (heidao 黑道), c'est-à-dire le monde du crime.

\section{La stratégie de traduction}

Quand Sellerio, maison d'édition palermitaine, commanda la traduction de Hong bai hei en 2015, le groupe était conscient d'introduire dans le marché italien un produit éditorial original, dont la nature hybride le différenciait sensiblement par rapport aux ouvrages littéraires chinois déjà disponibles en traduction italienne. Le choix de Sellerio s'appuyait sur des motivations spécifiques : d'abord, à côté de sa valeur littéraire, Hong bai hei possède une valeur documentaire importante susceptible de captiver le lectorat italien (et plus généralement européen), car le roman offre un regard littéraire privilégié sur les communautés chinoises à l'étranger et fait émerger la sensibilité propre d'un " traducteur culturel [...] négociant entre des champs expérientiels différents ${ }^{6} »$. Deuxièmement, d'un point de vue essentiellement économique, Sellerio qui en a acheté les droits mondiaux - visait expressément à la réalisation d'une traduction qui constituerait un point de repère pour d'autres éditeurs internationaux intéressés par son achat et sa publication, dans une perspective qui se voulait globalisante. Ces facteurs ont évidemment joué un rôle primordial aussi bien dans l'approche du texte de la part du traducteur que dans la formulation de la stratégie traduisante.

Sur le plan pratique, la stratégie adoptée dans la traduction de Hong bai hei s'est donc focalisée sur la conservation des expressions porteuses d'une connotation culturelle et spécifiques au contexte chinois, ainsi que sur la reconstruction - et même, dans maints cas, la recréation - de l'hétérolinguisme et, plus généralement, des éléments signalant l'altérité du texte. De plus, un travail d'interaction constante entre traducteur et auteur a permis d'adapter, voire reconstruire certains éléments du roman. Cet ensemble de stratégies, dans des formes et des mesures différentes, a ainsi contribué à redéfinir le « texte premier».

\section{Le traitement des realia et des expressions culturellement connotées}

Les realia - à savoir les mots dénotant des objets, des concepts ou des phénomènes propres à une culture donnée - ont été régulièrement transférés en pinyin (le système officiel de translittération du chinois mandarin), accompagné éventuellement d'une note en bas de page là où le contexte ne serait pas suffisant pour les rendre intelligibles. 
Il s'agit essentiellement de noms d'aliments (ex. mantou 馒头 " pain cuit à la vapeur non farci », baozi 包子 《pain cuit à la vapeur avec une farce à base de viande et/ou de légumes ») et d'unités de mesure (ex. cun 寸 « $3,3 \mathrm{~cm}$ », liang 两 = « $50 \mathrm{~g}$ », jin 斤 $=\ll 0,5 \mathrm{~kg} », e t c$.$) .$

Les références au contexte politique chinois, extrêmement fréquentes dans les chapitres se focalisant sur l'époque maoïste, méritent une mention particulière au sein de la catégorie des expressions porteuses d'une forte connotation culturelle. Ces expressions sont typiquement représentées par des titres et des appellations, souvent assez longs et complexes, ou par des slogans et des noms de campagnes politiques. Dans la première catégorie tombe p. ex. la qualification nüminbing lianzhang 女民兵连 长 ${ }^{7}$ " capitaine de la Compagnie de la Milice Populaire des Femmes ». Au deuxième groupe appartiennent des expressions telles que yi bang yi, yi dui hong 一帮一, 一对红 8 (litt. « un-aider-un, un-couple-rouge ») " si l'un aide l'autre, les deux deviennent rouges », qui se réfère à la pratique maoïste d'éduquer les élèves de l'école primaire à la conscience politique en les faisant travailler à côté de ceux issus d'une famille " rouge ", c'est-à-dire politiquement privilégiée ; ou encore san zhong ren 三种人 ${ }^{9}$ " les trois catégories ", objet d'une campagne lancée au début des années 80 contre les éléments radicaux considérés comme responsables des excès de la Révolution culturelle qui venait de s'achever. Dans tous ces cas, les expressions ont été reproduites littéralement, accompagnées par une note si nécessaire : loin de constituer un simple signal d'altérité, cette stratégie vise à révéler l'omniprésence de l'élément politique dans la société chinoise à l'ère maoïste et, en dernière analyse, à souligner le rôle joué par la « voie rouge » déjà évoquée dans la construction narrative.

\section{La reproduction et la recréation de I'hétérolinguisme}

Le traitement des éléments hétérolingues a comporté l'application d'une variété de microstratégies différentes. Dans le cas le plus simple - à savoir l'insertion, dans le texte chinois, de mots ou phrases en lettres latines, souvent accompagnés d'une traduction chinoise entre parenthèses - le traducteur a simplement transposé l'élément hétérolingue dans le texte traduit en le signalant par l'italique et en intervenant sur l'orthographe si nécessaire :

\section{人流不断在谢青身边走过, 好些人包括一些美丽的姑娘看到谢青时, 都会惊讶地 轻叫一: “KINAZI(中国人)”。 \\ Un flux incessant de promeneurs continuait à défiler devant Xie Qing : plusieurs d'entre eux, y compris des filles superbes, laissèrent échapper un «Kinezi ! » de surprise en le voyant.}

Dans cet extrait le mot albanais signifiant « chinois » a été reproduit en italique afin de le signaler en tant qu'élément hétérolingue, avec une correction orthographique de "Kinazi » à "Kinezi ${ }^{11}$. Le contexte et l'assonance permettent d'effacer la traduction du mot (présente entre parenthèses dans le texte chinois) sans en compromettre la compréhension.

L’identification des éléments hétérolingues s'avère plus problématique dans le cas de mots et d'expressions étrangers transcrits phonétiquement en caractères chinois, comme les suivants :

\footnotetext{
“西拉维斯! ”阿尔把尼亚人命令着谢青, 可他不明白这话的意思。后来的几天 里, 谢青经常听到阿尔巴尼亚人对他说这个词儿, 他环磨着可能意思是“不要乱 动” 12 。

«Mos u leviz! », lui ordonnèrent les Albanais, mais Xie Qing ne comprenait
} 
pas. Dans les jours qui suivirent, les entendant répéter cette phrase à plusieurs

reprises, il en conclut que cela devait signifier « Pas bouger ».

\section{他听到阿尔巴尼亚人说：易果！谢青知道“易果”是阿语“走”的意思 ${ }^{13}$ 。}

Il entendit les Albanais lui intimer : hikë ! Il savait que cela voulait dire « Cassetoi ! » en albanais.

Dans les deux premiers extraits, l'élément sémantique correspondant aux transcriptions phonétiques de l'albanais, à savoir xilaweisi西拉维斯 et yiguo 易果, a été identifié grâce aux annotations présentes dans le texte original. Toutefois, les formes chinoises étant transcrites de manière imparfaite, il a été nécessaire de remonter aux formes linguistiques albanaises les plus proches sur le plan sémantique, arrivant à " Mos u leviz ! » et " hikë» respectivement. Comme dans les cas évoqués auparavant, l'italique a été adopté afin de mieux signaler la composante hétérolingue ; de plus, le contexte rend toute explication supplémentaire superflue.

Le traducteur a opté pour une intervention plus active dans la recréation d'un hétérolinguisme inexistant dans l'original :

\section{小孩在喊：妈眯妈眯我回来了14！ \\ Le petit enfant se mit à crier : Mami, mami, me voilà ! \\ 然后谢青[...]听得阿尔巴尼亚人轻声喊着：一，二，三15！ \\ Xie Qing [...] entendit ensuite les Albanais compter à mi-voix : një, dy, tre !}

Dans le premier exemple, le mot mami 妈眯 (alternativement 妈咪) 《 maman », correspond fortuitement à la forme phonétique de son correspondant albanais mami. Le traducteur a décidé de le présenter comme un mot albanais à travers l'italique ; quant à la sémantique du mot, elle demeure transparente grâce à son affinité phonétique avec son traduisant dans la langue cible. Dans le deuxième exemple la phrase chinoise yi, er, san一, 二, 三 « un, deux, trois » a été traduite en albanais sans que la compréhension en soit compromise, en vertu de son affinité avec l'italien sur le plan phonétique et par la modulation du verbe han 喊 《crier » dans la forme « compter».

Des interventions localisées du même ordre ont été effectuées dans un certain nombre de noms d'activités commerciales. Par exemple, Haibin lüguan 海滨旅馆 ${ }^{16}$ « Hôtel front de mer » est devenu " Hôtel Bregdeti », où le mot albanais « bregdeti » signifie précisément « front de mer » et est couramment utilisé dans l'onomastique hôtelière albanaise. Ou encore, dans le cas du restaurant Fenghuanglou 凤凰楼 ${ }^{17}$ " Le Palais du phénix », le traducteur a opté pour la forme française "Le Phénix », un pastiche qui fait écho au nom de la célèbre librairie parisienne spécialiste de l'Asie.

Un cas particulier de recréation hétérolingue concerne le traitement du vocabulaire régional. Le texte original présente un nombre élevé de formes lexicales wenzhounaises qui ont pour fonction de marquer l'appartenance identitaire et manifestent ainsi un décalage - non seulement linguistique mais également culturel - entre le « centre » et une des «périphéries » chinoises. Dans certains cas le mot wenzhounais est compréhensible pour un lectorat chinois général en vertu de sa forme graphique en caractères chinois (bien que sa réalisation phonétique serait vraisemblablement incompréhensible pour un locuteur non natif) ; dans le pire des cas, sa fonction peut être déduite du contexte. Toutefois, puisque la plupart des éléments lexicaux s'avèrent particulièrement problématiques, une glose explicative est ajoutée par l'auteur.

Dans tous ces cas, le traducteur a opté pour une transcription de l'élément régional : cette dernière, pourtant, ne suit pas le pinyin mais, faute d'un autre système officiellement reconnu, les règles de romanisation du wenzhounais indiquées par la Société des Dialectes $\mathrm{Wu}^{18}$. Dans le cas des termes non annotés, la forme translittérée 
est suivie d'une traduction littérale en italien. Par exemple, l'appellation tongzilaor 童 子疼儿 ${ }^{19}$ (litt. 《enfant-tuberculeux ») - une épithète en apparence seulement péjorative utilisée en wenzhounais pour s'adresser à un enfant, employée par le maire d'AC dans ses conversations avec Xie Qing - a été transcrite dans la forme dontsyleong, suivie de la glose " petit tuberculeux ». Les expressions régionales déjà annotées dans l'original ont posé évidemment moins de problèmes, car la translittération ne nécessite aucune intervention supplémentaire. Ainsi, par exemple, le mot sannianbei 三年背 20 (litt. " trois-ans-dos ») " eucalyptus » a été transcrit dans la forme sanyipai ; c'est l'auteur même qui précise que ce nom vient du fait que le bois de l'eucalyptus peut être utilisé - après avoir été transporté sur le dos - lorsque la plante a atteint l'âge de trois ans. La même stratégie a été mise en place dans le traitement du mot pancai 盘菜 ${ }^{21}$ (litt. " plateau-légume »), dans la forme boetshe : le mot désigne une variété de navet mentionnée à plusieurs reprises dans le roman en vertu de son rôle primordial dans la cuisine et la culture wenzhounaises, qui en fait presque un symbole de l'identité locale.

\section{Une coopération auteur-traducteur pour recréer le texte premier}

Une partie substantielle du travail de traduction s'est appuyée sur une interaction constante avec l'auteur visant à « reconstruire » le texte " original ». Cette intervention s'est d'abord réalisée dans la réintégration d'un court passage (105 caractères au total), présent dans le manuscrit original mis à disposition du traducteur mais éliminé dans la version publiée par la maison d'édition chinoise Zuojia chubanshe, sans doute en raison des références sensibles à la réalité politique et aux pratiques policières chinoises :

\section{“我在那里躲了四个月, 到处都有记者和安全局的人在追堵我。而且我知道, 很 快就会有杀手来索我的命。因为我知道太多的事情, 国内一些要人怕我万一被引 渡回去对他们不利，一定会杀人灭口。所以，我用一个假护照逃到欧州来了 ${ }^{22}$ 。”}

« Je suis restée cachée là-bas pendant quatre mois, mais des journalistes et des gens du Ministère de la Sécurité d'Etat me poursuivaient partout. Je savais qu'un tueur me liquiderait bientôt. Je savais trop de choses, et l'idée qu'on puisse m'extrader en Chine faisait peur à certains gros bonnets, qui essaieraient sans aucun doute d'éliminer un témoin gênant. Je me suis donc réfugiée ici en Europe grâce à un faux passeport. »

De plus, sur requête de l'auteur, la traduction italienne comprend une section de l'épilogue (2077 caractères au total) qui ne paraît pas dans la version publiée et qui a été fournie directement au traducteur, afin de rendre la structure narrative plus accomplie $^{23}$.

À côté des ajouts textuels, l'interaction auteur-traducteur a également contribué à définir, de façon collaborative, une série de stratégies spécifiques visant à conserver la fonction documentaire de la traduction. Cette approche s'est concrétisée notamment dans la conservation des réflexions socio-culturelles sur le contexte non chinois et, de manière générale, des annotations essentiellement conçues pour le lectorat d'origine (la conversion des lires italiennes en dollars américains ${ }^{24}$, etc.). Au lieu de gommer des détails qui auraient été jugés superflus dans un projet de traduction familiarisante ${ }^{25}$, on a opté pour une opération de « défamiliarisation » des références familières au lectorat cible, afin de signaler encore une fois la présence constante d'un point de vue extérieur sur une réalité connue.

Ce processus de collaboration a également entraîné une série de révisions localisées du texte original, notamment la correction d'imprécisions évidentes : par exemple, le roman fait référence au quartier chinois de Belleville en le localisant de façon erronée dans le $13 \mathrm{e}$ arrondissement ${ }^{26}$, une imprécision corrigée dans la traduction en le repositionnant dans le 20e. C'est toujours suite aux échanges que j'ai eus avec Chen que 
certaines erreurs ont été délibérément sauvegardées pour des raisons liées à la construction narrative. C'est le cas, par exemple, de la référence implicite à Bule Naipi, héroïne de la résistance albanaise tuée par les occupants allemands en 1944 à l'âge de 22 ans. Toutefois, le roman situe sa mort en 1943, à 18 ans $^{27}$ : après une consultation avec l'auteur, le traducteur a été autorisé à corriger la date, tandis que la référence à l'âge a été gardée, bien qu'erronée, puisque ce détail était censé selon l'auteur augmenter la portée émotionnelle du passage ${ }^{28}$.

\section{Traduction, recréation, globalisation}

Les traits dominants du roman de Chen $\mathrm{He}$, ainsi que leurs implications sur la construction narrative, sont résumés efficacement par Luo :

\section{《红白黑》 [...]却有着不同于本土宏大叙事作品的“异质”。这一异质性, 最突出 地体现在作家对空间场景的调度与处理上。这使我们看到, 这部小说的背景渗透 了某种全球化的精神视野和多元文化的混杂性。这种创作手法突破了单一文化视 野的局限，使小说在思想意蕴与审美表达上获得了拓展和提升 ${ }^{29}$ 。}

Hong bai hei présente une " hétérogénéité " par rapport aux autres ouvrages chinois du grand récit. Cette hétérogénéité émerge notamment dans l'organisation et la gestion du scénario spatial par l'auteur : on peut remarquer que le roman se déroule sur une toile de fond imprégnée d'esprit de la globalisation et d'hybridité multiculturelle. Il s'agit là d'une technique de création qui dépasse les limites de la perspective monoculturelle, permettant au roman de développer et de renforcer ses implications idéologiques et son expression esthétique.

La réflexion sur le traitement de cette hétérogénéité radicale et la formulation de stratégies spécifiques ont forcément joué un rôle primordial dans le projet de traduction. En effet, la stratégie adoptée dans la traduction italienne de Hong bai hei est largement modelée sur une approche minorisante (minoritizing), pour utiliser la terminologie de Venuti. Puisque cette pratique « repose sur l'hétérogénéité discursive » et « résiste à l'éthique assimilatrice en signalant la différence linguistique et culturelle du texte ${ }^{30}$ », elle s'avère la plus appropriée pour faire émerger au niveau textuel le dynamisme linguistico-culturel et les composantes hétérogènes du roman.

La stratégie traduisante se concrétise d'abord dans une série de microstratégies lexicales, telle la reproduction des expressions porteuses d'une connotation culturelle, pour arriver au traitement des éléments linguistiques étrangers et régionaux et, finalement, à des interventions qui investissent le niveau proprement textuel remettant en question la notion même de texte premier.

Le choix de sauvegarder l'hétérolinguisme s'inscrit dans un projet visant à faire émerger en traduction, dans la mesure la plus ample possible, l'altérité constitutive du texte, ainsi que les tensions, les clivages et les contradictions externes et internes dont ce dernier est pénétré. Tous ces facteurs, qui se réalisent à plusieurs niveaux et sur des axes différents, sont véhiculés par la langue mais touchent à des niveaux beaucoup plus complexes. Par exemple, si les tensions liées aux différents scénarios spatiaux sont tout à fait évidentes, la mise en relief des régionalismes décrite plus haut vise à signaler un niveau de résistance plus profond. En fait, la traduction attribue aux éléments lexicaux du wenzhounais une proéminence, à la fois linguistique et graphique, que l'original ne manifeste pas, ou ne manifeste que dans une mesure limitée pour des raisons liées à la nature même de la langue chinoise écrite. Il s'agit là de souligner, avec une intensité encore plus prononcée que dans l'original, une contradiction culturelle interne entre centre et périphérie, entre culture dominante et marginale : en faisant remonter à la surface la composante minoritaire, le traducteur ne manifeste pas simplement une altérité par rapport à la dimension familière au lecteur cible, mais brise également l'homogénéité apparente de la «Chine » en mettant à l'épreuve l'expérience cognitive 
du destinataire du texte traduit. Cet effet est encore plus flagrant dans l'insertion originale du vocabulaire étranger (albanais, français, etc.) dans le texte traduit, qui vise à en intensifier la composante hétérolingue. Ce même effet de dépaysement passe par les procédés de "défamiliarisation " déjà évoqués à propos de la conservation des annotations socio-culturelles, qui soulignent la présence insistante d'un regard extérieur. Ces interventions relèvent incontestablement de la redéfinition, voire de la recréation, du texte premier : dans la perspective de la traduction comme copie originale, elles jouent donc un rôle complémentaire aux stratégies minorisantes visant à sauvegarder l'hétérogénéité textuelle.

Les ajouts textuels convenus avec l'auteur constituent un procédé qui remettent en cause la notion de texte premier de manière encore plus significative. La réintégration des détails politiquement sensibles répond premièrement à l'exigence, de la part de l'auteur, de rétablir des portions du texte qui n'avaient jamais vu le jour à cause de la censure (ou de l'autocensure). De plus, elle pourrait être interprétée comme un désir de mettre en évidence des éléments de critique politique qui s'avèrent attrayants pour les lecteurs non chinois - mais surtout pour les maisons d'édition étrangères qui s'aventurent dans le monde de la littérature chinoise contemporaine. L'ajout de l'épilogue, suggéré par Chen directement au traducteur, relève d'une exigence de nature plus strictement narrative : l'hypothèse que cette intégration réponde aux attentes et au goût d'un lecteur modèle étranger imaginé par l'auteur demeure pourtant fascinante. Il faut ajouter, à côté de ces facteurs, la nature du projet éditorial de Sellerio. Comme la maison palermitaine vise explicitement à réaliser une traduction qui servirait de modèle pour d'autres éditeurs internationaux, elle a déclaré son intention de réviser lourdement la traduction - en condensant ou éliminant, par exemple, les passages considérés comme verbeux ou non indispensables à la narration par les rédacteurs afin de produire un nouveau " texte premier », plus proche des attentes d'un lecteur « occidental » imaginé, prêt pour la promotion et l'exportation au-delà des frontières italiennes. Plus étonnant encore, Sellerio a également exprimé l'intention de restituer à l'auteur le texte résultant de cette révision pour qu'il l'adopte comme nouvel original. Il est évident qu'un tel projet présente des implications éthiques sérieuses, car il trahit une idée ethnocentrique qui se heurte violemment à la macrostratégie minorisante envisagée par le traducteur. Au-delà de ces dernières considérations, les opérations textuelles analysées ci-dessus signalent encore une fois les frontières floues du texte original, son identité toujours en cours de négociation, et finalement une osmose qui attribue à la traduction un rôle fortement créateur.

Cet ensemble complexe d'interventions mises en place à plusieurs niveaux et par une pluralité d'acteurs, peut effectivement produire un texte « qui dépasse les limites de la perspective monoculturelle », pour reprendre la définition de Luo. Toutefois, l'exacerbation des composantes hétérogènes ne doit pas forcément entraîner, chez le lecteur cible, l'illisibilité ou la frustration. En fait, « un emploi stratégique d'éléments minoritaires peut demeurer intelligible pour une vaste gamme de lecteurs ${ }^{31}$ », ne comportant qu'une interruption momentanée de la participation de ces derniers. Ces éléments potentiellement déstabilisateurs s'inscrivent dans un cadre esthétique et thématique qui n'est pas tout à fait éloigné de l'expérience du lecteur du texte traduit. Le destinataire de la traduction n'est donc pas confronté à un texte que la prépondérance d'éléments d'altérité rendrait entièrement dépaysant : il peut accueillir cette altérité et l'insérer en tant que telle au sein d'un cadre connu.

En dernière analyse, la nature " globalisée » de Hong bai hei appelle précisément à une approche de la traduction " globalisante ». Si le texte global met en relief « les états de clivage internes de la culture » et les processus de négociation continue qui soustendent la définition de l'identité culturelle, alors « les textes traduits peuvent [...] devenir un espace où des conflits linguistiques et identitaires sont mis en texte ${ }^{32}$ ». La traduction minorisante et la réécriture du texte premier deviennent ainsi les dispositifs qui permettent de faire émerger les traits d'un texte et d'un discours culturel qui, dans 
un contexte global, sont moins homogènes et monolithiques qu'on ne le supposerait ; et, en même temps, elle déclenche un court-circuit fructueux chez le lecteur, qui remet constamment en question sa lecture des frontières linguistiques et culturelles.

\section{Bibliographie}

Chen He 陈河, «Bei bangjiazhe shuo » 《被绑架者说》 (Le récit d'un kidnappé), Dangdai n ${ }^{\circ}$ 2, 2006, p. 163-189.

Chen He 陈河, Hong bai hei 红白黑 (Le Rouge, le Blanc et le Noir), Pékin, Zuojia chubanshe, 2012 [Il rosso, il bianco e il nero, trad. Paolo Magagnin, Palerme, Sellerio (sous presse)].

Feng Ping-chia, Diasporic Representations. Reading Chinese American Women's Fiction, Berlin, Lit Verlag, 2010.

Luo Yuhua 罗玉华, “'Hong bai hei' - Hongda xushi de ling yi zhong jiangou » 《红白黑》- 宏大叙事的另一种建构 (Le Rouge, le Blanc et le Noir - Un autre type de construction du grand récit), Wenyi bao, 24 août 2012, p. 4.

Meylaerts, Reine, "Les langues de la littérature : multilinguisme, traduction, identité », communication présentée à la Journée européenne des langues, Bruxelles, 26 septembre 2007.

Venuti, Lawrence, « Translation, Heterogeneity, Linguistics », TTR : Traduction, Terminologie, Rédaction, $\mathrm{n}^{\circ} 9$ (1), 1996, p. 91-115.

Venuti, Lawrence, The Scandals of Translation. Towards an Ethics of Difference, Londres et New York, Routledge, 1998.

Wuyu xiehui 吴语协会 (Société des Dialectes Wu), site consultée le 28 juin 2016, http://wuchinese.com.

\section{Notes}

1 Le choix de ce sigle, dû au désir de masquer les références sensibles à certains épisodes de la vie politique wenzhounaise, est purement fortuit et vient du nom de l'équipe de football italienne du Milan AC. Conversation privée avec Chen He, Pékin, 30 novembre 2015.

2 Ibid.

3 Chen He 陈河, Hong bai hei 红白黑 (《Le Rouge, le Blanc et le Noir »), Pékin, Zuojia chubanshe, 2012, p. 46. Pour des raisons pratiques, dans les exemples cités seule une version française est donnée qui représente une traduction littérale de l'italienne. Toutes les traductions françaises sont miennes.

4 Ibid., p. 137.

5 Cette dernière expérience fait l'objet également d'un long reportage. Chen He 陈河, «Bei bangjiazhe shuo » 被绑架者说 (Le récit d'un kidnappé), Dangdai n ${ }^{\circ}$ 2, 2006, p. 163-189.

6 Ping-chia Feng, Diasporic Representations. Reading Chinese American Women's Fiction, Berlin, Lit Verlag, 2010, p. 172.

7 Chen He, Hong bai hei, op. cit., p. 140.

8 Ibid., p. 22-23.

9 Ibid., p. 241.

10 Ibid., p. 114.

11 Je tiens à remercier Mlle Xhina Hilviu et M. Visar Sylaj pour leur aide dans l'identification et le traitement des références à la langue et au contexte albanais.

12 Chen He, Hong bai hei, op. cit., p. 163.

13 Ibid., p. 173-174.

14 Ibid., p. 160.

15 Ibid., p. 164.

16 Ibid., p. 159.

17 Ibid., p. 49.

18 Wuyu xiehui 吴语协会 (Société des Dialectes Wu), site consulté le 28 juin 2016, http://wuchinese.com. 
19 Chen He, Hong bai hei, op. cit., p. 237 sqq.

20 Ibid., p. 21.

21 Ibid., p. 255 sqq.

22 Message privé de l'auteur, 18 décembre 2015.

23 Ibid.

24 Chen He, Hong bai hei, op. cit., p. 200.

25 En effet, les indications de l'éditeur envisageaient un projet de traduction - et, dans un deuxième moment, de révision - où les détails " superflus » seraient éliminés et les passages « verbeux » abrégés.

26 Chen He, Hong bai hei, op. cit., p. 277.

27 Ibid., p. 133.

28 Conversation privée avec Chen He, Pékin, 30 novembre 2015.

29 Luo Yuhua 罗玉华, “'Hong bai hei’ - Hongda xushi de ling yi zhong jiangou 》《红白黑》 -一宏大叙事的另一种建构 (Le Rouge, le Blanc et le Noir - Un autre type de construction du grand récit), Wenyi bao, 24 août 2012, p. 4.

30 Lawrence Venuti, " Translation, Heterogeneity, Linguistics », TTR : Traduction, Terminologie, Rédaction, $\mathrm{n}^{\circ} 9$ (1), 1996, p. 94-95.

31 Lawrence Venuti, The Scandals of Translation. Towards an Ethics of Difference, Londres et New York, 1998, Routledge, p. 12.

32 Reine Meylaerts, "Les langues de la littérature : multilinguisme, traduction, identité », communication présentée à la Journée Européenne des Langues, Bruxelles, 26 septembre 2007.

\section{Pour citer cet article}

Référence électronique

Paolo Magagnin, « Traduire un texte entre les langues. Retour sur l'expérience de traduction de Hong bai hei », TRANS- [En ligne], | 2017, mis en ligne le 27 septembre 2017, consulté le 26 novembre 2017. URL : http://trans.revues.org/1630 ; DOI : 10.4000/trans.1630

\section{Auteur}

\section{Paolo Magagnin}

Paolo Magagnin est professeur adjoint (ricercatore a tempo determinato) de langue chinoise à I'Université Ca' Foscari de Venise, ainsi que membre correspondant de l'UMR IrAsia (CNRS/Université Aix-Marseille). Son travail de recherche porte notamment sur la littérature chinoise moderne et contemporaine, la traductologie, l'enseignement de la littérature et de la culture chinoise et le discours politique chinois contemporain. II a également traduit en italien plusieurs ouvrages d'auteurs chinois contemporains tels que Zhu Wen, Xiao Bai, Xu Zechen, Chen He, Cao Wenxuan et A Yi.

\section{Droits d'auteur}

Tous droits réservés 